\title{
Multi-channel, robust measurement system for efficient flight testing
}

\author{
Kai Gilbert A. Author \\ imc Test \& Measurement GmbH, Max-Planck-Str. 22b, 61381 Friedrichsdorf/Ts., Germany \\ kai.gilbert@imc-frankfurt.de
}

\begin{abstract}
With the increasing complexity of the latest types of aircraft, the requirements placed on today's flight test systems have also increased. A wide variety of signals and sensors, as well as an ever-increasing channel count from digital sources and aircraft buses, are acquired over multiple channels, monitored online and, ideally, synchronously stored in a common data sink. The constantly demanding time schedules coming from management require a particularly stabile and effective FTI system. In this case, it is a great advantage to be able to obtain all system components from a single source. For a solution to this challenge, imc Meßsysteme $\mathrm{GmbH}$ has put together a modular flight test system based on imc measurement systems and video technology. All acquired analog and digital values are stored in the aircraft and transmitted in parallel to the control station by PCM. Thus, at any given time, it is possible to view and evaluate the measurement data via imc STUDIO Monitor without having to land the aircraft. This saves time. Additionally, imc supports the instrumentation and commissioning when necessary with their own personnel resources to reach the goal faster.
\end{abstract}

Key words: flight test, data acquisition, telemetry, monitoring, robust measurement system

\section{Intro}

Before a new type of aircraft can be put into production, an extensive program for testing and certification must be passed. Performance testing, safety testing and low- and hightemperature tests are just a few of the requirements for certification (Certification flight tests).

\section{High demands placed on data acquisition}

For flight testing, prototype aircraft are equipped with comprehensive test flight instrumentation.

A variety of signals and sensors must be synchronously acquired. These include, for example:

- Strains

- Accelerations

- Temperatures

- Positions

- Pressures

- Voltages

- Displacements
In addition to analog sensors, these days a large amount of information communicated in digital form via buses like:

- ARINC

- ASCB-D

- CAN

- RS232, RS422, RS485

- Ethernet

- USB, etc.

In order to obtain visual information and cockpit displays parallel with the measurement data, synchronous video recordings from freelypositionable cameras are necessary.

In total, the test instrumentation in a single aircraft can range from a few hundred up to several thousand analog and digital measurement signals. All of these data are time-synchronously acquired in the aircraft and safely recorded in a central data pool. This saves from having subsequent combinations of different data sources. This results in time savings when supplying the measurement data for subsequent analyses. 


\section{Telemetric signal transmission}

All test flights are typically coordinated and monitored by test engineers in a control room located back on the ground. To give them the capability to make the best possible assessment of the situation at any given time, telemetric transmission of selected measurement signals from the instrumented prototype aircraft to the control room is necessary. The transmitted data can then be monitored and saved online in the control room at the various monitor positions.

\section{Modern flight test system}

For such complex flight test instrumentations, the test and measurement specialists at imc Meßsysteme $\mathrm{GmbH}$ offer a modern solution concept. Thanks to its modular design, the imc flight test system can be optimally adapted to meet aircraft-specific tasks and flexibly expanded to meet future requirements as well.

The imc flight test system is based on:

1.) Modular imc measurement systems for all analog and digital data sources

2.) imc video technology with up to 8 cameras featuring individual resolution and frame rate settings

3.) Control PC with monitor

4.) Airworthy storage, network and server technology

5.) Telemetric signal transmission via PCM

\section{Universal and modular measurement hardware}

The foundation of the data acquisition is built with robust and airworthy imc measurement systems. With a modular design and universal measurement amplifier technology, a tailored layout is possible for capturing signals. Depending on the scope of the instrumentation, multiple measurement systems can be used and networked allowing thousands of channels to be synchronously acquired. The system can either be centrally installed in a 19" rack or spatially distributed throughout the aircraft. The measurement systems capture all analog and digital measurement values, as well as ARINC and ASCB-D field bus data. What is particularly helpful is that the measurement devices provide direct, real-time calculations. This delivers results during the running measurement and helps to reduce data down to the essentials.

\section{- IIIIIIIII - IIIIIIII!}

Fig. 1. imc CRONOScompact modular measurement system

\section{Flexible video technology}

To be able to associate measurement data and flight information, time-synchronous video is necessary. For this, the video stream of the cockpit displays, as well as freely-positionable video cameras, are connected over a DVI matrix. This distributes the video information to monitors within the aircraft and to other highperformance PCs that can synchronously record the measurement data with the video data.

\section{The heart of the test set-up}

The testing is configured via a fanless, highperformance PC which serves as a control computer. Test engineers use this computer to transfer the configuration created in the office to the flight test system and make any adjustments when necessary. In addition to the configuration, the control computer also collects and centrally stores all of the measurement data from the different measurement systems. Simultaneously, the same measurement data stand available for online visualization on the monitors within the aircraft.

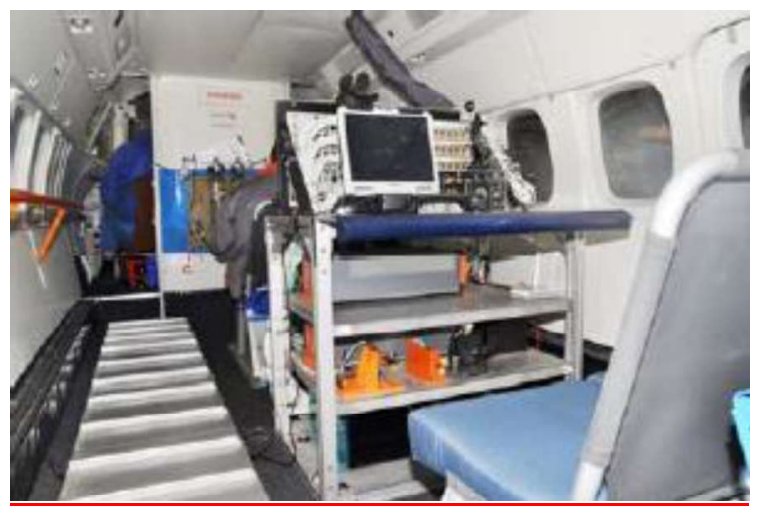

Fig. 2. Flight test system with monitor position in a test aircraft

\section{Secure, central data storage}

In the configuration software, the user decides where the data is to be stored.

- Internally on the measurement system's CF card

- On the control PC 
- $\quad$ On a robust NAS drive (MIL-STD-810E)

- Redundant measurement data storage

The NAS drive allows for one-handed operation of a removable storage medium which will enable a quick transfer of the measurement data to the control room. At the local ground station server, the transferred data can be immediately reproduced, evaluated and analyzed.



Fig. 3. imc flight test system design within an aircraft

\section{Stabile power supply}

To stabilize the power supply, the imc flight test system is equipped with a UPS system. This buffers temporary voltage drops and allows depending on the batteries used - a selfsufficient operational time from 30 minutes up to several hours.

\section{Telemetric signal transmission saves time}

With a click of a mouse button, test engineers can select a telemetric transmission of all of the acquired signals within the aircraft. By means of PCM (pulse-code modulation), the selected measurement data will be transmitted online to the control center on the ground where they can be decoded, displayed and saved. This way, test engineers and technicians can already view and evaluate data and statuses online during the actual test flight. Of course, a real-time display of the measurement data is possible in the aircraft itself as well as in the control room this flexibility is important to perform the testing in the most time-efficient manner as possible. Because the telemetric transmission is limited by your bandwidth, separate sampling rates can be configured on a channel-wise basis for the PCM transmission.

\section{Comfortable software with flight-specific display elements}

The complete operation and display of the measurement is carried out with imc STUDIO measurement software. It allows for convenient configuring of the test set-up from the office PC without being connected with the systems. In addition, it offers numerous possibilities for the visualization of the measurement data. For example, position data can be displayed on a moving map that literally moves in the curve window in relation to the position of the test aircraft.

With the imc Export Program, the user is able to carry out subsequent analyses of the measurement data with their own evaluation software. Alternatively, the powerful imc 
FAMOS analysis software is also available. In addition to numerous display options and hundreds of ready-made analysis functions, it also offers automated data evaluations and provides a simple means of creating professional reports, thus increasing productivity.

\section{Conclusion}

Modern aircraft developments require increasingly complex testing within a tight timeframe. With the imc flight test system, test engineers have a proven and flexible solution available to them. All relevant measurement values from the flight test, such as that from sensors, aircraft buses, cockpit displays and video feeds, can be acquired synchronously from multiple channels and securely stored.

The comfortable software shortens configuration times and provides visualization and analysis at the push of a button. Furthermore, telemetric signal transmission allows more testing to be conducted in a shorter amount of time because the ground personnel can make direct assessments of the measurements without requiring the test aircraft to land between tests. This saves time and ensures productive test sequences without stopping.

imc Meßsysteme $\mathrm{GmbH}$ provides continued support to their customers with comprehensive services, such as with instrumentation, signal connections, commissioning or evaluations.

\section{Lessons learned}

Because of the multiple data sources and thousands of measurement channels, one of the greatest challenges turns out to be the startup time of the complete system.

In order to record all of the different data sources together in one data sink, it requires a lot of effort to safely boot up all of the components and have stabile operation with each other.

In the case of the imc flight test system, a configurable startup sequencer is used that guarantees an orderly startup of individual hardware components, thus ensuring a safe startup of the entire system.

As a further challenge, operation of the system is performed by various users who operate their individual measurement PCs differently. This leads to changes in software and firmware statuses that can cause problems upon startup of the system.

The solution to this is a fixed boot manager that resets all PCs upon startup to a defined starting configuration. This requires, however, that the boot time is extended by approx. 1 $\min 45 \mathrm{sec}$.

Since it is desired that all data sources are synchronously recorded, an external timecode interface is used which synchronizes all system components to a single time base. This process extends the startup time by an additional 2 minutes.

After the synchronization, the software configuration and the experiment are loaded into the connected hardware components and the system is prepared for startup. This process takes an additional 3 min $30 \mathrm{sec}$ for all systems. Loading of the UMS data (digital data source with approx. 3000 channels) appeared to be the only outlier and significantly delayed the startup time of the overall system. This operation requires up to 8 minutes.

This puts the total startup time for the system at 12 minutes.

In conclusion, the following can be said: if startup times less than 5 minutes are required, then it will only be possible by sacrificing functionality.

For example, if synchronous video recording is omitted, then all of the measurement data can be locally saved in the imc measurement systems. Thus, additional measurement and storage PCs are obsolete. Such a configuration has the big advantage of being extremely stabile (imc standard software) and is ready to calculate within a short startup time of approx. 2 minutes. The disadvantage is that the data must be transferred after the measurement via a transfer program to an evaluation PC, or as an alternative, the flash cards in the measurement systems must be exchanged. 